\title{
Intranuclear Coccidiosis in a Calf
}

\author{
Tomoko NISHIDA ${ }^{1)}$, Shinichi HATAMA ${ }^{2)}$, Yoshiharu ISHIKAWA ${ }^{2)}$ and Koichi KADOTA ${ }^{2) *}$ \\ I) Iburi Livestock Hygiene Service Center, 4-3 Tomiura, Noboribetsu 059-0462 and \\ ${ }^{2)}$ Hokkaido Research Station, National Institute of Animal Health, 4 Hitsujigaoka, Toyohira, Sapporo 062-0045, Japan
}

(Received 9 January 2009/Accepted 7 April 2009)

\begin{abstract}
A case of intranuclear coccidiosis in an 11-month-old female Japanese Black calf is described. Because the calf exhibited diarrhea and failure to grow and then became moribund, euthanasia was performed. Histologically, the small intestinal villi were highly atrophic, and the epithelial cells frequently had meronts or merozoites within the nuclei. A few intranuclear gametocytes were also seen. The presence of Eimeria alabamensis-specific DNA in the lesions was demonstrated by polymerase chain reaction and nucleotide sequence analyses. Electron microscopy disclosed that most parasites were within nuclear inclusions encircled by the two membranes derived from the nuclear envelope, and that intracytoplasmic meronts and merozoites were in direct contact with the cytosol. Apparently, mature merozoites could be free without destruction of the host cells, and this may be associated with the relatively low pathogenicity of this coccidium.
\end{abstract}

KEY WORDS: cattle, Eimeria alabamensis, intranuclear coccidiosis.

J. Vet. Med. Sci. 71(8): 1109-1113, 2009

Intranuclear coccidiosis has been reported chiefly in birds [6], tortoises [7] and lizards [5], and also in amphibians [12] and fish [14]. Although extremely rare in mammals, a few instances of intranuclear infection have been reported: Eimeria (E.) alabamensis in cattle [3,4] and Cyclospora (C.) talpae in moles [11].

In tortoises, intranuclear coccidia are capable of inducing death of the hosts [7]. Reports of other reptilian hosts, however, did not make reference to the pathogenicity, and intranuclear coccidia are detectable in some captive geckoes [5]. In geese, E. stigmosa [6] and E. hermani [15] were considered to be specialized parasites that have evolved to live within their hosts in intimate states of symbiosis. E. alabamensis causes disease at extremely large infective doses only $[3,8]$. Clinical outbreaks have been reported from some European countries [2], and dehydration, depression and reduced growth may be seen in calves kept on heavily contaminated pasture [19]. This paper reports a case of intranuclear coccidiosis in an 11-month-old female Japanese Black calf with diarrhea. The parasite, which had $E$. alabamensis-specific DNA, was characterized by its localization within nuclear inclusions surrounded by the two membranes derived from the nuclear envelope.

An 11-month-old female Japanese Black calf, which was presented with a 3-week history of anorexia and watery, greenish-brown diarrhea, showed severe failure to grow and rough hair coat. There were no oocysts of Eimeria in fecal samples. The condition did not improve despite treatment with fluids and gastrointestinal protectant. The calf became moribund and was euthanized 5 days after the first examination. At necropsy, the mucosa of the jejunum and ileum was thickened mildly.

\footnotetext{
* Correspondence to: Kadota, K., Hokkaido Research Station, National Institute of Animal Health, 4 Hitsujigaoka, Toyohira, Sapporo 062-0045, Japan. e-mail:kkadota@affrc.go.jp
}

Tissues were fixed in $10 \%$ buffered formalin, embedded in paraffin, sectioned at $4 \mu \mathrm{m}$, and stained with hematoxylin and eosin (HE), Giemsa, and naphthol AS-D chloroacetate esterase (CAE). Immunohistochemistry was performed by the streptoavidin-biotin complex/horseradish peroxidase (SAB-PO) method on histologic sections using Histofine SAB-PO kits (Nichirei, Tokyo, Japan). The primary antibodies utilized were mouse monoclonal antibodies to HAM56 for human macrophages (Dako, Carpinteria, CA, U.S.A.), CD79a (Dako, Glostrup, Denmark) and WC1-N3 (Veterinary Medical Research and Development, Pullman, WA, U.S.A.), and a rabbit polyclonal antibody to CD3 (Dako, Glostrup, Denmark). Small pieces of formalin-fixed tissues were post-fixed in $1 \%$ osmium tetroxide, and embedded in epoxy resin. Ultrathin sections were stained with uranyl acetate and lead citrate, and examined by electron microscopy (EM).

DNAs were extracted from paraffin sections by the phenol-chloroform-isoamyl alcohol method as described previously [9], and used as templates for polymerase chain reaction (PCR). For PCR amplification Ei18Sf1 forward (5'-AGCTCGTAGTTGGATTTCTG-3') and Ei18Sr1 reverse (5'-CTAGTCGGCATAGTTTATGG-3') primers were used. Both primers were designed based on the nucleotide sequence identity from relatively conserved regions in the $18 \mathrm{~S}$ ribosomal RNA coding region among previously described Eimeria spp. Amplification was done using ExTaq DNA polymerase (Takara Shuzo, Kyoto, Japan) according to the manufacturer's instructions. Amplicons were separated on $2.0 \%$ agarose gels, stained with ethidium bromide and detected by UV transillumination.

The PCR products were gel purified using the QIAquick Gel Extraction Kit (Qiagen, Hilden, Germany) and direct sequencing was performed with forward and reverse primers using the ABI PRISM Big Dye Terminator cycle sequencing kit (Applied Biosystems, CA, U.S.A.) and an 


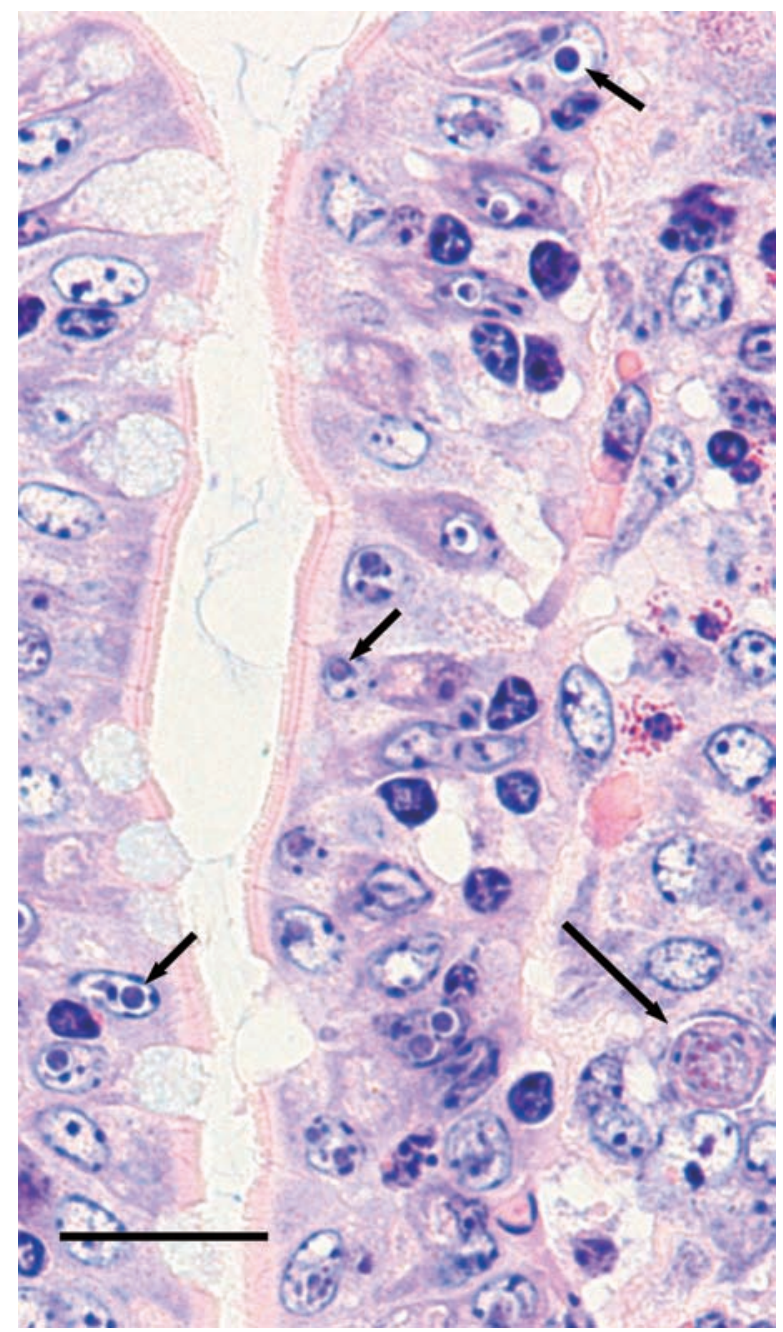

Fig. 1. Intranuclear meronts (short arrows) predominate in this field. An epithelial cell with a macrogametocyte (long arrow) is visible in the cytoplasm of an epithelioid cell. Giemsa. Bar $=20$ $\mu \mathrm{m}$.

ABI 3130 genetic analyzer (Applied Biosystems). The forward and reverse complementary sequences were aligned using GENETYX computer software (version 13). The nucleotide sequences obtained were compared to homologous sequences of previously characterized Eimeria spp.

Histologically, the jejunal and ileal villi were highly atrophic, but the crypts were elongated. The villous epithelial cells, most of which were neither necrotic nor apoptotic, often contained meronts or merozoites within the nuclei (Fig. 1), and less frequently within the cytoplasm. There were a few intranuclear macrogametocytes (Fig. 2) and microgametocytes, and no oocysts. Giemsa stain was more appropriate than HE stain in detecting parasites in tissue sections. Irregularly contoured nuclei were observed not only in epithelial cells with intracytoplasmic merozoites but also in cells with no parasites. There were a large number of intraepithelial lymphocytes, some of which contained eosi-

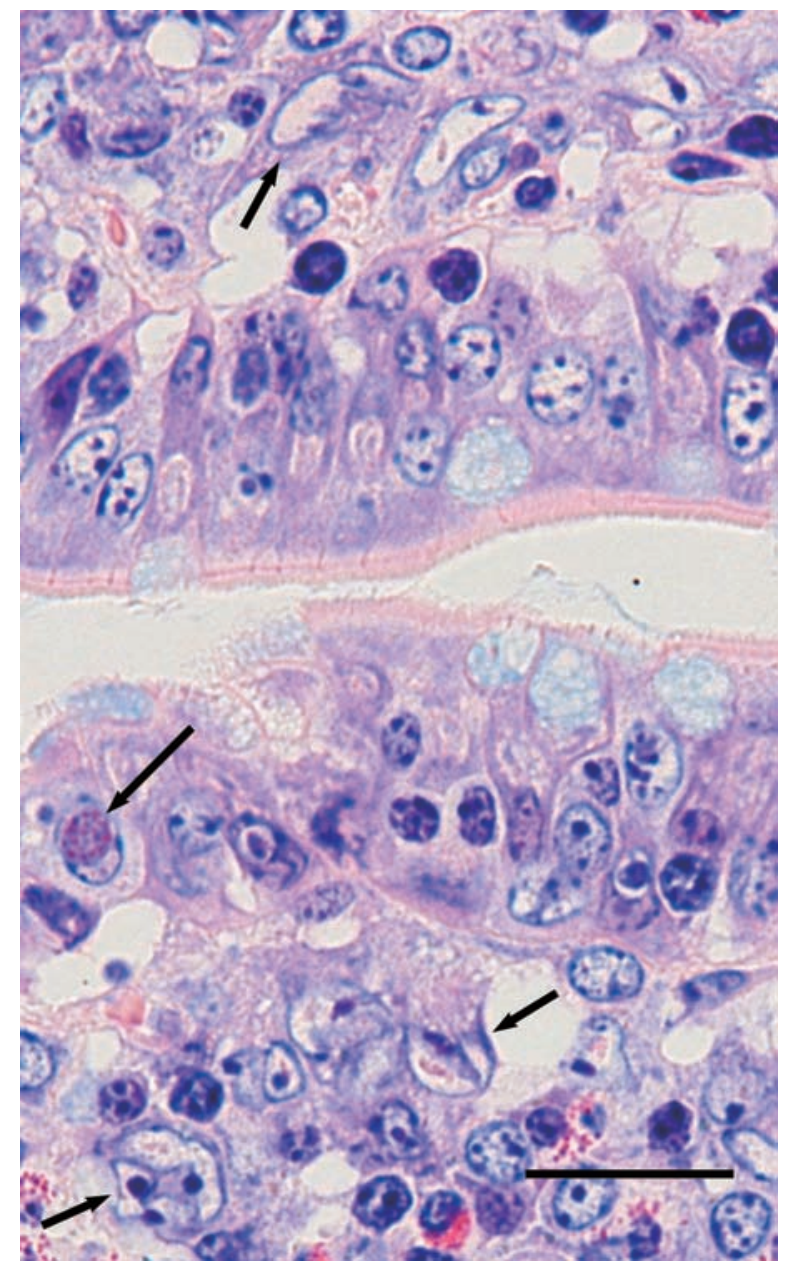

Fig. 2. Short arrows indicate epithelioid cells with highly irregular nuclei. An epithelial cell has a nucleus containing a macrogametocyte (long arrow). Giemsa. Bar $=20 \mu \mathrm{m}$.

nophilic granules positive for CAE in the cytoplasm. Macrophages and epithelioid cells frequently with irregular or multiple nuclei accumulated beneath the villous epithelium (Fig. 2), and the latter cells rarely phagocytosed merozoites, epithelial cells containing a macrogametocyte (Fig. 1), or cellular debris. Examination of the large intestine revealed no abnormalities.

Immunohistochemically, nearly all intraepithelial lymphocytes were $\mathrm{CD} 3$ positive, and some were $\mathrm{WC} 1$ positive. Most macrophages and epithelioid cells were stained with monoclonal antibody HAM56.

Meronts, merozoites and macrogametocytes could be observed by EM (Fig. 3). Intranuclear parasites were present as nuclear inclusions bounded by the two membranes derived from the nuclear envelope, and condensed chromatin was visible close to the outer membrane (Fig. 4). In the inclusions, the parasites were surrounded by host cell 


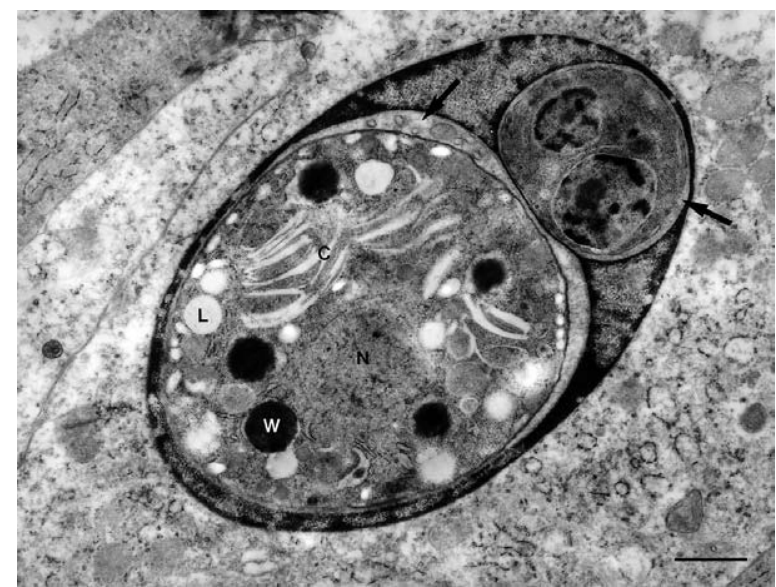

Fig. 3. A macrogametocyte and a binuclear meront are encircled by a narrow rim of host cell cytoplasm (arrows). Abbreviations: C, canaliculi; L, lipid droplets, N, nuclei; W, wall forming bodies. EM. Bar $=1.5 \mu \mathrm{m}$.

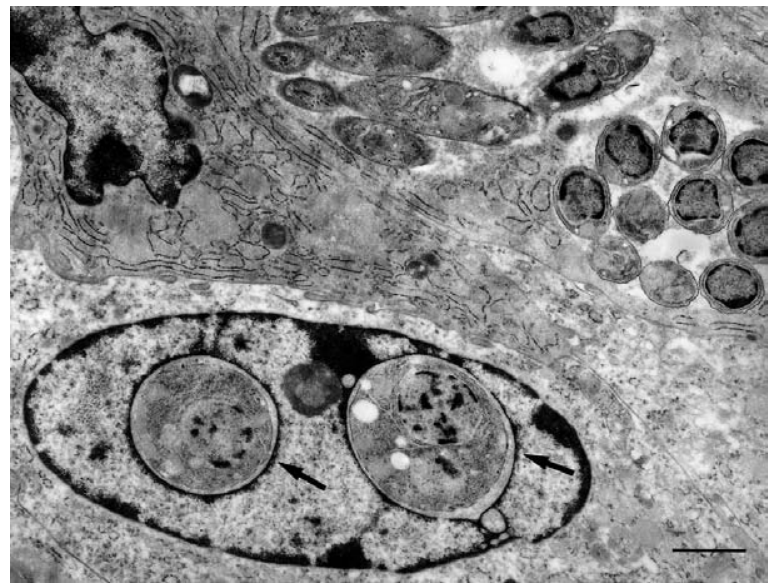

Fig. 4. Condensed chromatin is seen lying around the membrane of 2 nuclear inclusions (arrows), though it is less than marginated heterochromatin. There are no membranes around intracytoplasmic merozoites (upper right). EM. Bar $=2 \mu \mathrm{m}$.

cytoplasmic material (Fig. 3), but only small amounts surrounded mature merozoites (Fig. 5). In the host cell cytoplasm, immature meronts and mature merozoites were in direct contact with the cytosol (Fig. 4), and some of the former, which were located within invaginations of the nuclear envelope (Fig. 6), appeared to be at the transitional stage into intranuclear coccidia. Some epithelial cells without parasites possessed deeply invaginated nuclear profiles (Fig. 5).

A DNA fragment was amplified from the small intestine specimen containing a histologically disordered area by PCR analysis (Fig. 7). No DNA fragment was amplified from the large intestine, lung, liver, heart or cerebrum samples. The amplified DNA was further identified by nucleotide sequence analysis. It comprises $421 \mathrm{bp}$ in length and corresponds perfectly to the homologous sequence in the

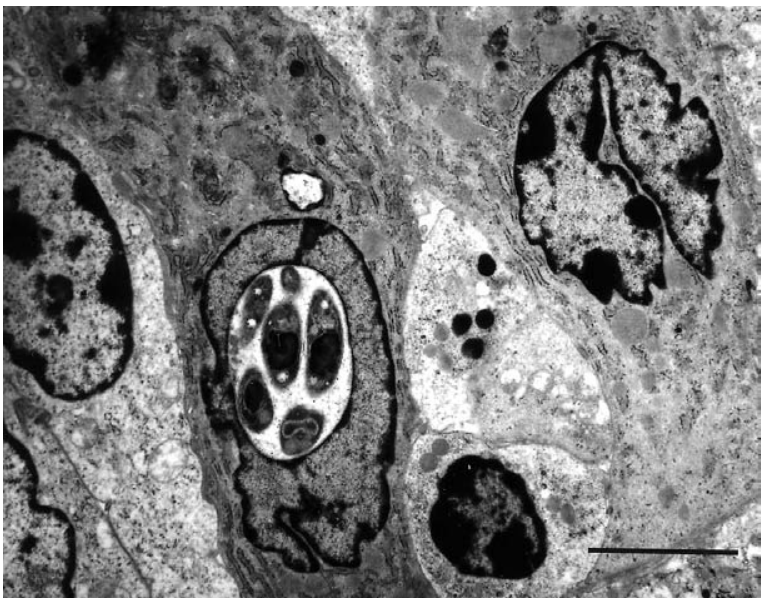

Fig. 5. Mature merozoites appear to be present within an intranuclear vacuole. An epithelial cell has a deeply cleaved nucleus (upper right). EM. Bar $=4 \mu \mathrm{m}$.

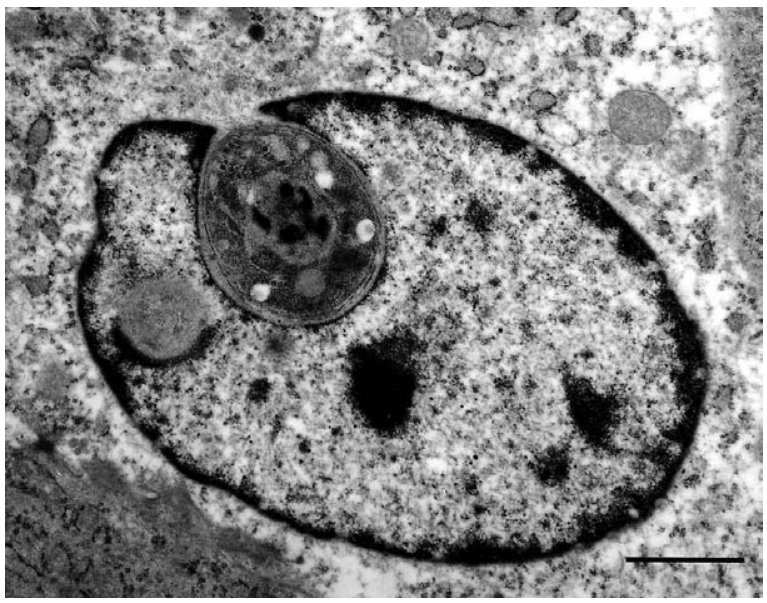

Fig. 6. An invagination of the nuclear envelope contains a meront. EM. Bar $=2 \mu \mathrm{m}$.

genome of E. alabamensis (Genbank accession number AF291427).

In mammals, intranuclear coccidiosis has been reported in moles [11] and cattle [3]. Most intranuclear coccidia including $C$. talpae in moles are unrelated to pathologic lesions, whereas E. alabamensis can induce watery diarrhea without blood in calves [2]. The clinical and histopathologic features in the current case were reminiscent of $E$. alabamensis infection, and the PCR examination established the diagnosis of chronic enteritis brought about by E. alabamensis. The negative result for oocysts in fecal samples, which is a reflection of their absence in tissue sections, indicates that fecal examinations may be useless at later stages of $E$. alabamensis infection.

In a study of $E$. hermani in geese, all of the developing stages were located within parasitophorous vacuoles bordered with a single unit membrane, whose presence was also 


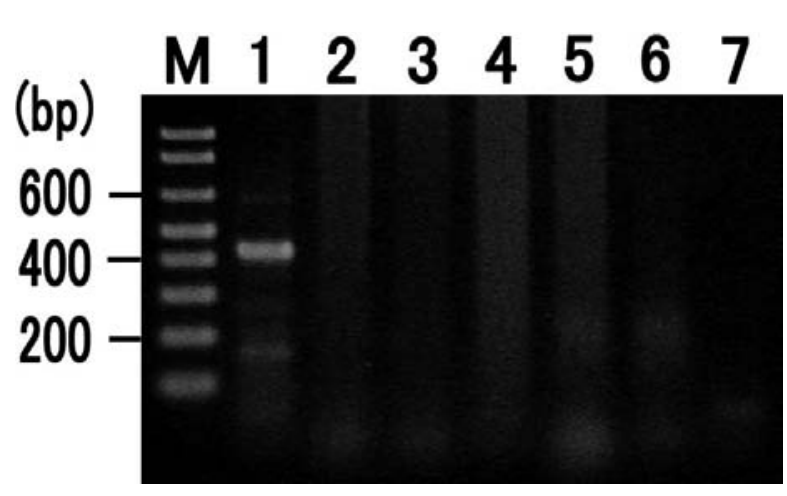

Fig. 7. PCR analysis of Eimeria $18 \mathrm{~S}$ ribosomal RNA coding region. M: 100 bp DNA ladder, lane 1: small intestine, lane 2: large intestine, lane 3: lung, lane 4: liver, lane 5: heart, lane 6: cerebrum, 7: negative control (distilled water).

demonstrated in most of the other intranuclear coccidia [15]. In contrast, Gajadhar et al. [6] observed the development of endogenous stages of E. stigmosa in close apposition with the host cell nuclear matrix. In mammalian cell cultures, intranuclear meronts of E. alabamensis were observed within parasitophorous vacuoles [18]. In the current ultrastructural study, however, most parasites were present as nuclear inclusions bounded by the two membranes derived from invaginations of the nuclear envelope, and the paucity of host cell cytoplasmic material around mature merozoites was thought to be evidence of its ingestion by the parasites [16]. In the cytoplasm of cultured cells, meronts were enclosed within parasitophorous vacuoles [17], whereas in the present case intracytoplasmic meronts and merozoites were in direct contact with the cytosol. Thus, there were considerable differences between the findings from in vitro and in vivo studies.

As merozoites of E. alabamensis escape from the nuclei of cultured cells, the nuclear membrane disintegrates [18]. In the current case, however, epithelial cells with or without intracytoplasmic merozoites frequently had intact but irregularly contoured nuclei. This implies that merozoites can escape from the host cell nuclei or cytoplasm without inducing their destruction, and that inclusions containing mature merozoites fuse with the nuclear envelope to become invaginations. These phenomena may be associated with the lower pathogenicity than that of highly pathogenic coccidia such as E. bovis and E. zuernii. Relatively severe diarrhea in the calf was thought to be due to the existence of many oocysts in the environment, because E. alabamensis may be highly pathogenic when large numbers of oocysts are given [3].

In the current case, there were increased numbers of intraepithelial lymphocytes and fair numbers of epithelioid cells. Some of the former were considered to be CAE-positive large granular lymphocytes or WC1-positive $\gamma \delta$ T cells, because CAE and WC1 have been demonstrated in bovine large granular lymphocyte lymphoma [13] and epitheliotropic $\gamma \delta \mathrm{T}$ cell lymphoma, respectively [10]. The latter were positive for HAM56, which is a marker for human and bovine macrophages [1]. Since the number of gametocytes was far smaller than that of meronts and merozoites, these inflammatory cells were thought to be reactive chiefly to extracellular merozoites, which were observed in the previous study [4] but not in the present study. Thus, the epithelioid cells just beneath the epithelium played an important role in host defense against the parasites, but may have prevented the absorption of nutritional substances.

\section{REFERENCES}

1. Anjiki, T., Wada, Y., Honma, H., Niizeki, H., Shibahara, T. and Kadota, K. 2000. Malignant histiocytosis in cattle. J. Vet. Med. Sci. 62: 1235-1240.

2. Daugschies, A. and Najdrowski, M. 2005. Eimeriosis in cattle: current understanding. J. Vet. Med. B 52: 417-427.

3. Davis, L. R., Boughton, D. C. and Bowman, G. W. 1955. Biology and pathogenicity of Eimeria alabamensis Christensen, 1941, an intranuclear coccidium of cattle. Am. J. Vet. Res. 16: 274-281.

4. Davis, L. R., Bowman, G. W. and Boughton, D. C. 1957. The endogenous development of Eimeria alabamensis Christensen, 1941, an intranuclear coccidium of cattle. J. Protozool. 4: 225229.

5. Finkelman, S. and Paperna, I. 2002. The endogenous development of four new species of Isospora Schneider, 1881 (Apicomplexa: Eimeriidae) from Australian geckos. Syst. Parasitol. 51: 59-71.

6. Gajadhar, A. A., Rainnie, D. J. and Cawthorn, R. J. 1986. Description of the goose coccidium Eimeria stigmosa (Klimes, 1963), with evidence of intranuclear development. J. Parasitol. 72: $588-594$.

7. Garner, M. M., Gardiner, C. H., Wellehan, J. F. X., Johnson, A. J., McNamara, T., Linn, M., Terrell, S. P., Childress, A. and Jacobson, E. R. 2006. Intranuclear coccidiosis in tortoises: nine cases. Vet. Pathol. 43: 311-320.

8. Hooshmad-Rad, P., Svensson, C. and Uggla, A. 1994. Experimental Eimeria alabamensis infection in calves. Vet. Parasitol. 53: $23-32$.

9. Imai, K., Mase M., Yamaguchi, S., Yuasa, N. and Nakamura, K. 1998. Detection of chicken anaemia virus DNA from formalin-fixed tissues by polymerase chain reaction. Res. Vet. Sci. 64: 205-208.

10. Kadota, K., Wada, Y., Ishikawa, Y. and Shibahara, T. 2001. $\gamma \delta$ T-cell lymphoma with tropism for various types of epithelium in a cow. J. Comp. Pathol. 124: 308-312.

11. Mohamed, H. A. and Molyneux, D. H. 1990. Developmental stages of Cyclospora talpae in the liver and bile duct of the mole (Talpa europaea). Parasitology 101: 345-350.

12. Noller, W. 1923. Zur Kenntnis einem Nierencoccids. Der Entwicklungskreis des Coccids der Wasserfroschniere (Isospora lieberkuhni). Archiv Protist. 47: 101-108.

13. Nozaki, S., Sasaki K., Ando, M. and Kadota, K. 2006. Natural killer-like T-cell lymphoma in a calf. J. Comp. Pathol. 135: $47-51$.

14. Paperna, I. 1995. Ultrastructural and developmental affinities of piscine coccidia. Dis. Aqua. Organ. 22: 67-76.

15. Pecka, Z. 1993. Intranuclear development of asexual and sexual generations of Eimeria hermani Farr, 1953, the coccidial parasite of geese. Zentralbl. Bakteriol. 278: 570-576.

16. Sampson, J. R. and Hammond, D. M. 1971. Ingestion of host- 
cell cytoplasm by micropores in Eimeria alabamensis. J. Parasitol. 57: 1133-1135.

17. Sampson, J. R. and Hammond, D. M. 1972. Fine structural aspects of development of Eimeria alabamensis schizonts in cell cultures. J. Parasitol. 58: 311-322.

18. Sampson, J. R., Hammond, D. M. and Ernst, J. V. 1971. Devel- opment of Eimeria alabamensis from cattle in mammalian cell cultures. J. Protozool. 18: 120-128.

19. Svensson, C., Uggla, A. and Pehrson, B. 1994. Eimeria alabamensis infection as a cause of diarrhoea in calves at pasture. Vet. Parasitol. 53: 33-43. 